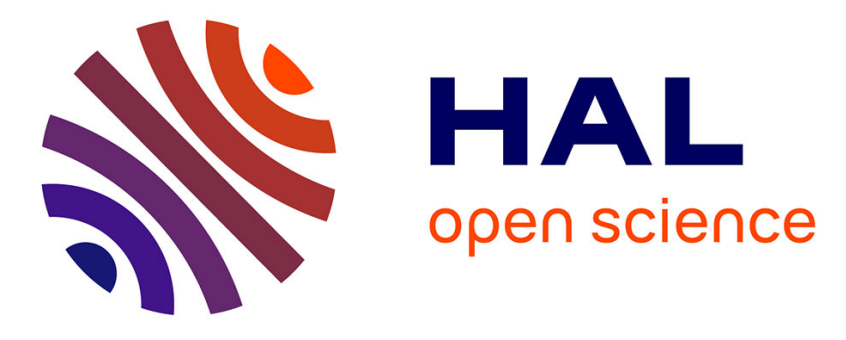

\title{
Temporal Deep Learning for Drone Micro-Doppler Classification
}

Daniel Brooks, Olivier Schwander, Frédéric Barbaresco, Jean-Yves Schneider, Matthieu Cord

\section{- To cite this version:}

Daniel Brooks, Olivier Schwander, Frédéric Barbaresco, Jean-Yves Schneider, Matthieu Cord. Temporal Deep Learning for Drone Micro-Doppler Classification. IRS 2018 - 19th International Radar Symposium, Jun 2018, Bonn, Germany. 10.23919/IRS.2018.8447963 . hal-02290839

\section{HAL Id: hal-02290839 \\ https://hal.science/hal-02290839}

Submitted on 18 Sep 2019

HAL is a multi-disciplinary open access archive for the deposit and dissemination of scientific research documents, whether they are published or not. The documents may come from teaching and research institutions in France or abroad, or from public or private research centers.
L'archive ouverte pluridisciplinaire HAL, est destinée au dépôt et à la diffusion de documents scientifiques de niveau recherche, publiés ou non, émanant des établissements d'enseignement et de recherche français ou étrangers, des laboratoires publics ou privés. 


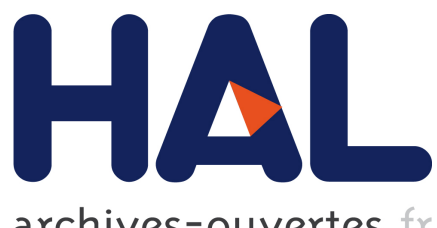

archives-ouvertes

\section{Temporal Deep Learning for Drone Micro-Doppler Classification}

Thales Limours, Daniel Brooks, Olivier Schwander, Frédéric Barbaresco, Jean-Yves Schneider, Matthieu Cord

\section{- To cite this version:}

Thales Limours, Daniel Brooks, Olivier Schwander, Frédéric Barbaresco, Jean-Yves Schneider, et al.. Temporal Deep Learning for Drone Micro-Doppler Classification. 2018 19th International Radar Symposium (IRS), Jun 2018, Bonn, France. pp.1-10. hal-02290839

\section{HAL Id: hal-02290839 \\ https://hal.archives-ouvertes.fr/hal-02290839}

Submitted on 18 Sep 2019

HAL is a multi-disciplinary open access archive for the deposit and dissemination of scientific research documents, whether they are published or not. The documents may come from teaching and research institutions in France or abroad, or from public or private research centers.
L'archive ouverte pluridisciplinaire HAL, est destinée au dépôt et à la diffusion de documents scientifiques de niveau recherche, publiés ou non, émanant des établissements d'enseignement et de recherche français ou étrangers, des laboratoires publics ou privés. 


\title{
Temporal Deep Learning for Drone Micro-Doppler Classification
}

\author{
Daniel Brooks $^{* \dagger}$, Olivier Schwander ${ }^{\dagger}$, Frederic Barbaresco*, Jean-Yves Schneider*, \\ Matthieu Cord ${ }^{\dagger}$
}

\author{
${ }^{*}$ Thales \\ Limours, FRANCE \\ email: daniel.brooks@lip6.fr \\ ${ }^{\dagger}$ LIP6 \\ Paris, FRANCE
}

\begin{abstract}
:
Our work builds temporal deep learning architectures for the classification of time-frequency signal representations on a novel model of simulated radar datasets. We show and compare the success of these models and validate the interest of temporal structures to gain on classification confidence over time.
\end{abstract}

\section{Introduction}

With the popularization of ever-more diverse and miniaturized Unmanned Aircraft Vehicles (UAVs, commonly known as drones), keeping an updated knowledge of airspace occupation has recently evolved to a much more complex challenge. Modern targets require finer analysis, for instance exploiting micro-Doppler signatures [5]. As an illustration, figure 1 shows the three drones we aim at classifying: The Vario helicopter and DJI's Phantom2 and S1000+. The task considers surface radars aiming at UAVs, be it for counter-UAV military applications or civil Unmanned Aircraft Systems (UAS) traffic management applications.

Previous work on radar classification with classical [9] [15] or deep learning techniques explore fully-connected or convolutional classification architectures. Our work also builds on deep learning but focusses on modern techniques adapted to capture temporal fluctuation information, which we consider a key feature in radar signals as it is in [18]. Lack of much real data inspires building simulations to emulate data at will, in order to gain in expressivity. Moreover, the proliferation of UAV models begs for generic and flexible modelling of intra and inter-class variations, key to building powerful learning models further on. In section 2 we describe a rather simple yet expressive model, which amounts to the paper's first contribution. The second is the comparison of two deep learning architectures on classification, related in section 3 . Section 4 describes experiments to validate both contributions and is followed by the conclusion. 

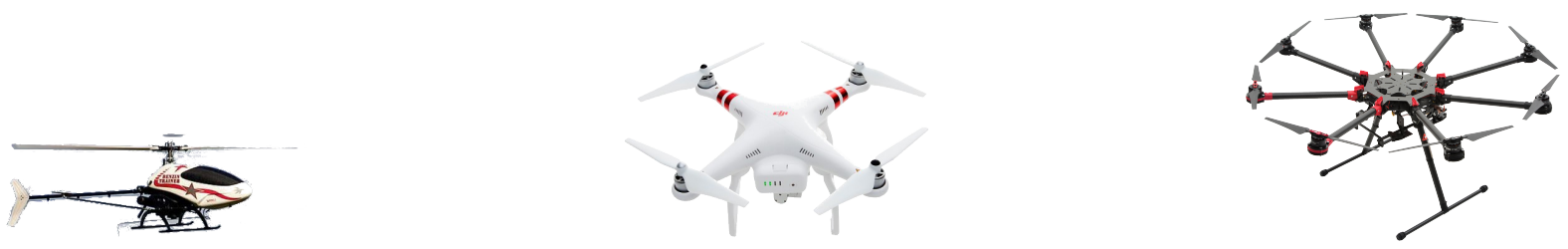

Figure 1: From left to right: The Vario helicopter, DJI's Phantom2 and S1000+. Scale is not respected.

\section{Radar Signal and Simulation}

In its raw form, a radar signal is the result of an emitted wave reflected off a target, sampled at a given pulse repetition frequency $(P R F)$, which yields a numerical time series of complex points (amplitude and phase) of intrinsic time-frequency nature [4]. Figure 2 shows how a mere fixed-time spectrum already allows for human interpretation and important feature extraction such as blade rotation speed $\Omega=\frac{R P M}{60}$ expressed in rad.s $\mathrm{s}^{-1}$, Radar Cross-Section (RCS), number $N_{b}$ and length $L_{b}$ of blades and radial velocity $V_{r}$. However, the extraction will likely not be robust to real-world variations, thus nor will any subsequent classification algorithm.

\subsection{A Physical Drone Model}

In this section we introduce a simple yet expressive drone simulator. Firstly, the UAV is modelled by a discrete set of $N_{p}$ scattering points disseminated along its physical structure. Figure 3 shows the distribution of the scattering points, their reflection direction and $R C S$ along with their temporal evolution. Note the model is 2-D as we consider the UAV to remain roughly in the same plane wrt the radar. Slight changes in inclination can be modelled by variations in the $R C S$. Future work may include simulating roll, yaw and pitch and corresponding individual blade rotation speeds.

The $N_{p}$ scattering points moving in time yield a set of $N_{p}$ series of 2-D coordinates, which are then fed to wave equations which return a temporal series of complex points. Finally, the signal is immersed in a noisy and cluttered environment. The noise is unavoidable white thermic noise from the sensing mechanism, the clutter sums up the influence the outside environment on the signal of interest; Billingsley's model was used to model ground clutter [2]. The following section extends the simulator to account for unpredictible behavior, thereby introducing intraclass variety.

\subsection{Dataset Generation}

The simulator described above gives a deterministic output given one configuration. Real data are however subject to multiple variations even within one single measurement, whether it be responses to an unkown controller, alterations of the environment or the data acquisition itself, 


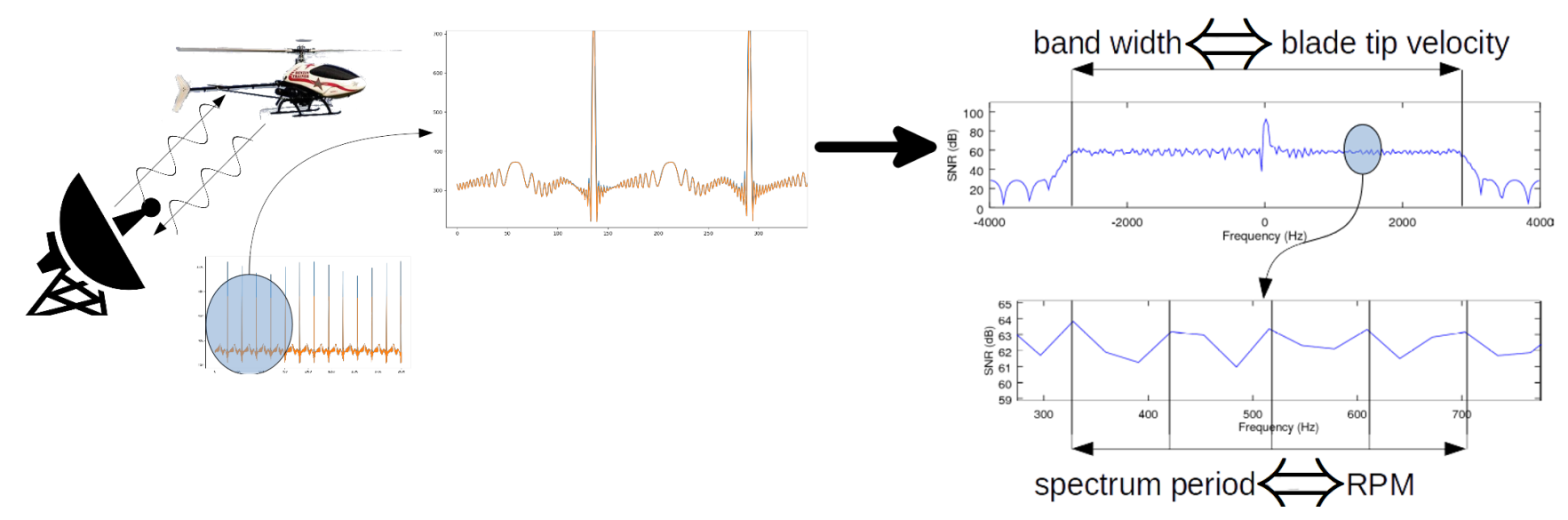

Figure 2: Illustration of the simulated signal. Here, a $P R F$ of $8 k H z$ on a signal of $250 \mathrm{~ms}$ yields a time series of 2000 complex points, jointly drawn as modulus and real part. From a simple analysis of the featured spectrum, we can deduce three important characteristics of the UAV: rotation speed $\Omega$, number $N_{b}$ and length $L_{b}$ of blades. To the left: zoom-in of the original signal $(S N R=50 d B)$. Top right: complete Fourier transform $(P R F=8 k H z)$, where we define $B$ as the signal's bandwidth (the plateau in the spectrum). Bottom right: zoom-in of the spectrum, where we define $\nu$ the observed period of its assumed periodicity. Then we have $\nu=N_{b} \Omega$ and $B=\frac{4 L_{b} \Omega f_{e}}{c}$, which gives access to the three unkowns up to a hypothesis on the number of blades, clean analysis pending. Note that for the sake of clear figures, the signal representation parameters such as as the $P R F$ and the signal to noise ratio $(S N R)$ were set to wishfully accomodating values. It is also important to point out that a gain in $S N R$ of $10 \log \left(n_{f f t}\right) \approx$ $15 d B$ is achieved in performing the Fourier transform. The given $S N R$ values take that gain into account.
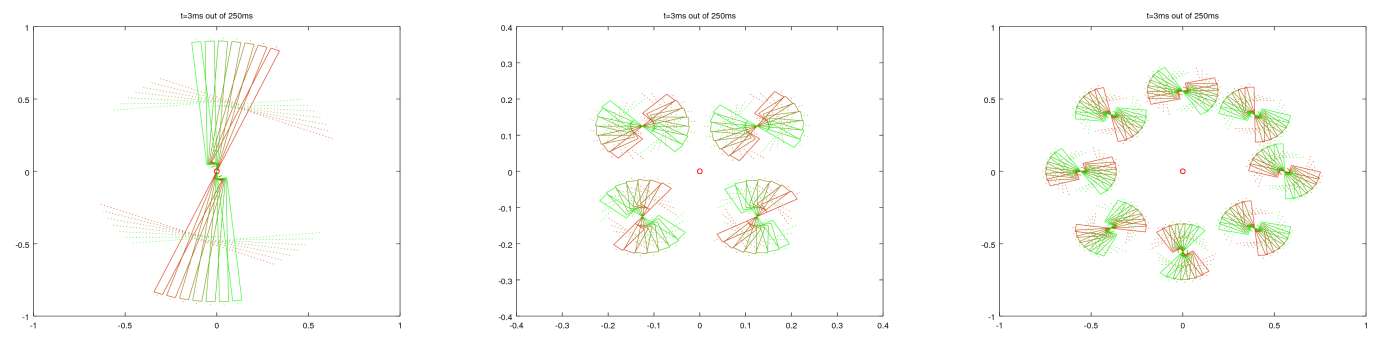

Figure 3: Evolution of the scattering points and normals for the Vario, Phantom2 and S1000+ drones for a total simulation time of $250 \mathrm{~ms}$, of which we only illustrate the first $3 \mathrm{~ms}$ (corresponding to 7 frames) for the sake of visual simplicity. The body is modelled as an isotropic reflector whereas blades are scattered with directional punctual reflectors in the direction of the normals, the length of which are proportional to the points' reflectance. Notice the multicopters' helices alternatively turn clockwise and counter-clockwise. The 2-D scale is in meters; dimensions and distances are accurate wrt reality. Time goes from red to green. 


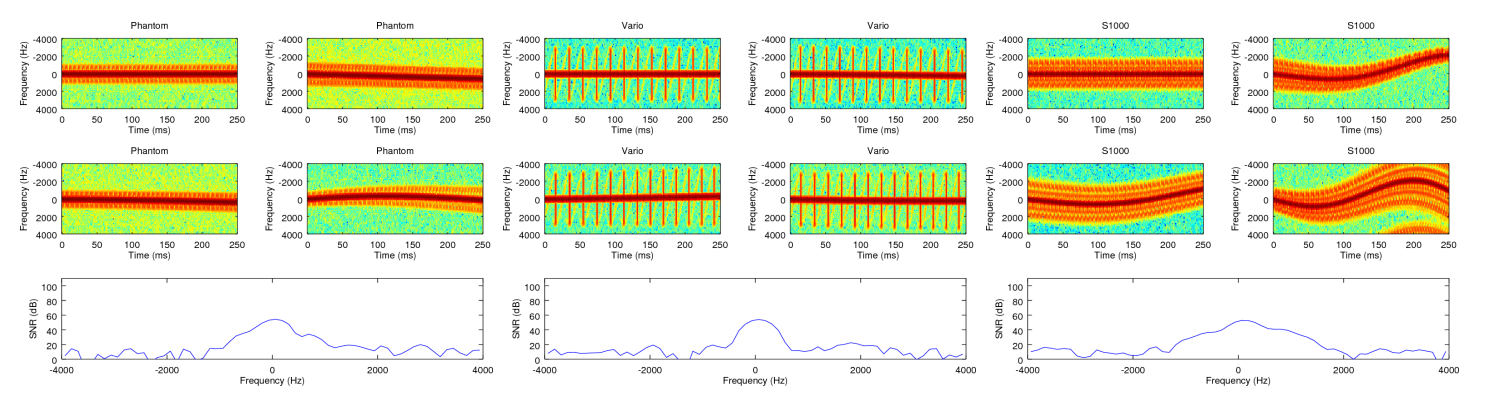

Figure 4: Spectrograms of noisy and uncluttered signals for the three drones mentioned above, from left to right: the Vario, the Phantom2 and the S1000+. Each group is organized as follows: four varying spectrograms of the same class are displayed; the top-left one always corresponds to a version constant in time. Below we plot an arbitrary time cut of one of the varying spectrograms. Important parameters of the representation are: $S N R=40 \mathrm{~dB}, P R F=8 \mathrm{kHz}$, Simulation time $T=250 \mathrm{~ms}, N_{f f t}=64$ points and window overlap percentage $p_{o v}=50 \%$. Again, we take into account the gain in the Fourier transform and chose very forgiving configuration parameters for the sake of visual clarity.

all in all to non-cooperative behavior. These variations will define the intra-class disparity and thus the inter-class separability. We have chosen four parameters to model disparity: $R C S, V_{r}$, $\Omega$ and flight curvature $\kappa$. Their values are found or estimated from drone specifications while their variations still need to be heuristically estimated in order to allow for more expressive sampling. For instance, the $R P M \mathrm{~s}$ in ${\mathrm{rad} . \mathrm{s}^{-1}}$ are constrained as follows:

1. Vario: $R P M \in[1550 ; 1650]$

2. Phantom: $R P M \in[4250 ; 5750]$

3. $\mathrm{S} 1000+: R P M \in[3250 ; 4750]$

Figure 4 shows intra and inter-class variations for the three drones Vario, Phantom 2 and S1000+ for a given set of representation parameters. Intra-class variety is achieved by sampling pairs of drone parameter values in their acceptable ranges and linearly interpolating the resulting values in time. Visible temporal variations are as accurate as possible, although exaggerated, wrt reality. They exhibit discriminative behaviours which we hope to capture in learning algorithms. The reader may observe that the S1000+, under strong variations, bypasses the allowed bandwidth set by the $P R F$, which leads to the well known frequency folding phenomenon [19], or Doppler ambiguity, which in turn can hurt the representationss credibility as a robust one. Unfortunately, though we can set the parameter arbitrarily high in simulations (again, which we do here for the sake of visual clarity), real-world radar costs constrain the $P R F$ to possibly sub-optimal thresholds. 


\section{Deep learning on radar signals}

This section gives an overview of the base concepts of statistical learning developed in our classification algorithms. The simplest form of such algorithms is linear classification such as logistic regression [8], a stacking of which amounts to the simplest form of neural networks, the multi-layer perceptron (MLP) [16]. Contrary to MLPs, convolutional neural networks (CNNs, first introduced in [13] and popularized in [12]) exhibit shared and locally connected filters, ie convolutions, to exploit spatial locality in images.

\subsection{Fully-convolutional networks for signal classification}

Seeing a time-frequency representation as an image to be analyzed via a CNN constitutes a first attempt at deep radar classification. One first subtlety to handle is that choosing the same representation for input signals of potentially different lengths will lead to frequency dilution. The more natural solution is to use the same projection instead of the same representation, eg a spectrogam of fixed window size and overlap. This of course allows input data of different dimensions, not easily handled by standard CNNs. A natural bypass is to consider the problem as Multiple Instace Learning [20], or MIL. In this framework, one input data point is seen as a collection of multiple atomic instances of the same class. Concretely, we divide spectrogams in segments of equal length, which we denote as $\tau$. We define these segments as noisemes (in analogy to phonemes in speech recognition). The choice of $\tau$ is rather arbitrary, and is interpreted as the minimal duration one must observe the signal to accurately determine its class.

There exists a modification of CNNs which, although seemingly trivial, allows for semantic segmentation, ie pixel-level, or, in the case of radar classification, timestep-level classification: Fully-convolutional networks (FCNs), introduced in 2016 by [14], where dense layers of size $n$ are replaced with convolutional layers of size 1 and depth $n$. This artificial transformation is referred to in the paper as the "convolutionalization trick". Additionally, a Global Average (or Max) Pooling (GAP) layer ensures correct dimensionality before final classification. Importantly, the neural network now naturally deals with inputs of varying size and outputs temporal semantic feature maps when fed with longer signals. It is also worth noting the computational advantage of an FCN over, say, a sweeping of the original CNN on patches of data: indeed the architecture is highly optimized for parallel computation, which amounts to high training and testing speed on GPU, which allows for a variety of challenging applications such as image segmentation [10]. We design such a network, which we may name temporal fully-convolutional network or TFCN, illustrated in figure 5.

The choice of hyperparameters for the network directly influences the inherent temporal precision achieved by the TFCN, in particular the filter and pooling sizes and associated strides. Since the FCN is built upon the CNN classifier, the temporal feature map length $l_{o}$ equals 1 when given an input of length $l_{i}=\tau$. We note $\mathcal{S}\left(l_{i}\right):=l_{o}$ the global sizing function of the network, composed of $L$ layer-wise such functions $\mathcal{S}_{l}$ such that $S_{l}(n):=n_{l+1}=\left\lfloor\frac{n_{l}-k_{l}+2 p_{l}}{s_{l}}\right\rfloor+1$, 


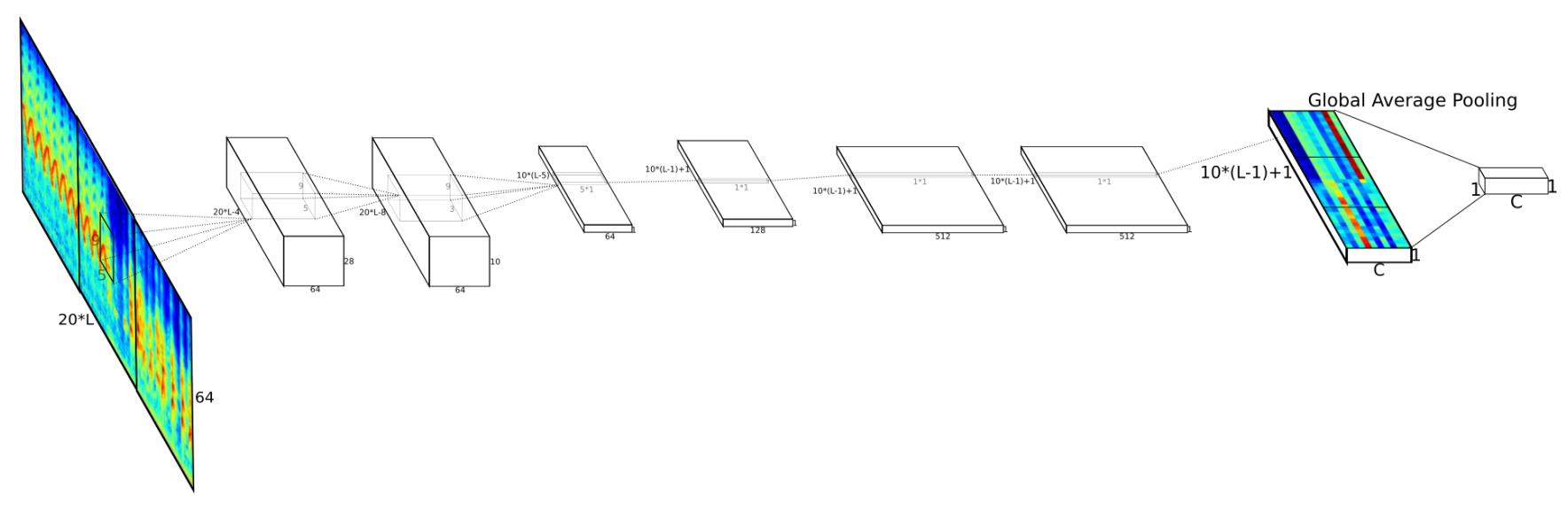

Figure 5: The proposed architecture for radar signal segmentation, where the boxes represent the successive filter banks. Horizontal is time, vertical is frequency. $\mathrm{C}$ is the number of classes, ie 3 if we consider the Vario, Phantom 2 and S1000+. In this example we feature a similar 10-class problem which has more visual interest. Contrary to Computer Vision, where the feature maps are spatial, our feature maps extend solely in time. We fix the noiseme length $\tau$ to 20 timesteps. Note that we omit pooling layers, strides, batchnorms, dropouts and non-linearities in the figure for visual simplicity.

where $n_{l}$ and $n_{l+1}$ are the input and output size at layer $l$ and $k_{l}, p_{l}$ and $s_{l}$ respectively the filter size, pad and stride at layer $l$. In the case of the proposed architecture 5, we have:

$$
\forall k \in \mathbb{N}^{*}, \mathcal{S}(k \tau)=\mathcal{S}_{L} \circ \cdots \circ \mathcal{S}_{1}(k \tau)=\left\lfloor\frac{5}{2} k \tau-\frac{5}{2}\right\rfloor+1:=\lfloor\mathfrak{f}(k-1) \tau\rfloor+1
$$

We introduced in the above equation 1 the finesse coefficient $\mathfrak{f}$ which quantifies the superresolution reached within a noiseme. By construction, $\mathfrak{f} \in[1 ; \tau]$, and its explicit formula is simply $\mathfrak{f}=\left(\prod_{l=1}^{L} s_{l}\right)^{-1}$ (again, $s_{l}$ being the stride at layer $l$ ). For instance with $\tau=20$ and $\mathfrak{f}=\frac{5}{2}$, we can classify the signal every 8 timesteps instead of 20 , ie perform classification of controled precision. Overlapping receptive fields are one way to do so, we will see in the next section how neural networks can inherently support time dependency.

\subsection{Recurrent neural networks for signal classification}

Recurrent neural networks (RNNs) originally stem from standard perceptrons, but loop the inner states to learn on sequences of data rather than on individual, unordered points [7]. The core equation for RNNs is quite similar to that of perceptrons for it only adds the hidden state time dependency. RNNs do not naturally handle out time-frequency images as they are vector-based. Nonetheless, by considering the spectrogam no longer as an image but rather as a series of 1-D spectrums, which in essence it is, we face no more concern. In the recurrent framework, the finesse coefficient $\mathfrak{f}$ is equal to its maximal value $\tau$ as we are dealing with individual spectrums. 
We can nonetheless relax $\mathfrak{f}$ by adding a stride in the sequential spectrums, which amounts to subsampling the original time-frequency representation. In practice we implement a two-layer more sophisticated version of recurrent networks, Long Short Term Memory networks [11] (LSTM) which are able to learn longer time dependecies [3] and seem robust to slight time warpings in input signals [21], with 256 units at each layer.

\section{Experiments}

In this section we validate experimentally the classification of simulated data and study the influence of important parameters. To achieve this we build several datasets corresponding to different configurations.

\subsection{Experimental setup}

All datasets contain $N=1000$ examples for each of the $C=3$ classes, of which we reserve $25 \%$ for testing. We perform a 64-point sliding Fourier transform with Hamming windowing. We choose the noiseme length $\tau=20$ spectrogram timesteps, which corresponds to $\approx 15 \mathrm{~ms}$ in real life (the choice is rather arbitrary though guided by the largest approximate period in all signals). Throughout the experiments we train the three classifiers (MLP, RNN and FCN) with the same strategy to remain consistent: stochastic gradient descent (SGD) with initial learning rate $\alpha=0.5$ for the MLP, RMSProp [17] with $\alpha=2 e-3$ and decay $\beta=1 e-6$ for the RNN, and accelerated SGD with $\alpha=4 e-3$ and momentum $\mu=0.9$ for the FCN. All optimizations are initialized by Glorot uniform sampling and run for $K=100$ epochs without early stopping nor cross-validation (except manual hyper-parameter finetuning). All learning rates are divided by 2 every 25 epochs. Models were implemented using the Keras [6] library with Tensorflow [1] backend and trained on a single Nvidia GTX 1070M GPU. Training a model takes less than an hour with all models.

\subsection{General performance and robustness}

First we train the classifiers multiple times on a standard configuration to evaluate overall performance and robustness to initialization to evaluate the simulator's expressivity, seen in figure 6 . Running the training 20 times for each classifier (we actually test three FCN architectures with different finesse coefficients), we observe consistent accuracy results, the most consistent being the FCN, which exhibits the smallest spread. In terms of accuracy, the MLP falls way behind the RNN and FCNs, which are close though the FCNs seem to generally perform better.

\subsection{Impact of radar configuration parameters}

Here we train all models in different configurations; specifically we vary $S N R$ and $P R F$ from extremely challenging to wishfully accomodating. Results are found in figure 7 . The most chal- 

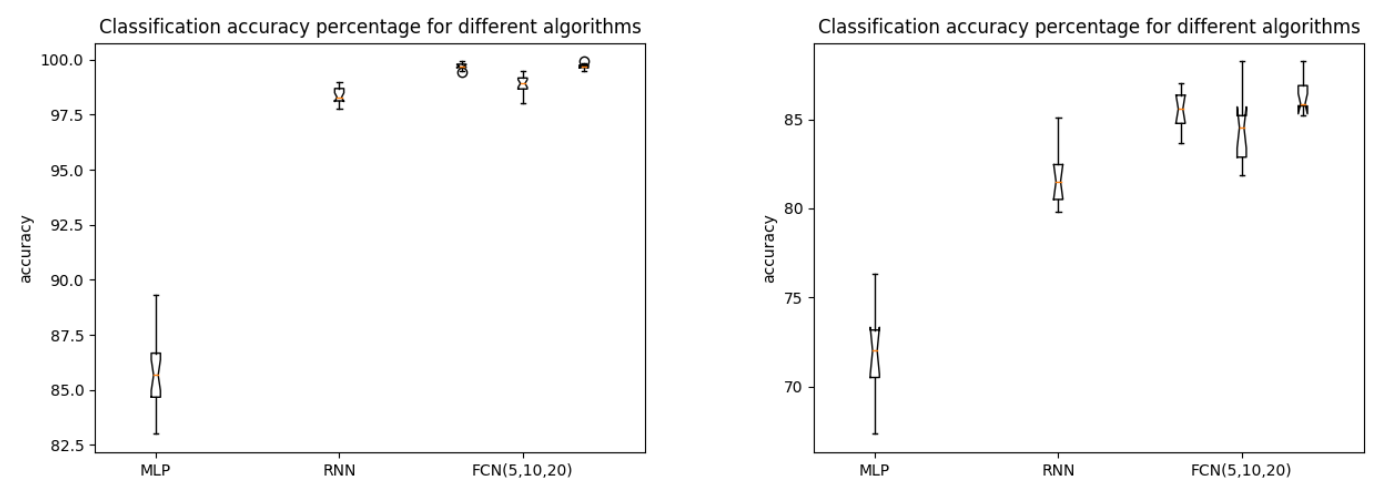

Figure 6: Classification accuracies for three learning models. Here we chose a setup of cluttered, noisy signals with $S N R=30 \mathrm{~dB}$ on the left and $S N R=10 \mathrm{~dB}$ to the right $(P R F=4 k \mathrm{~Hz})$, which is more than reasonable from a practicioner's point of view.
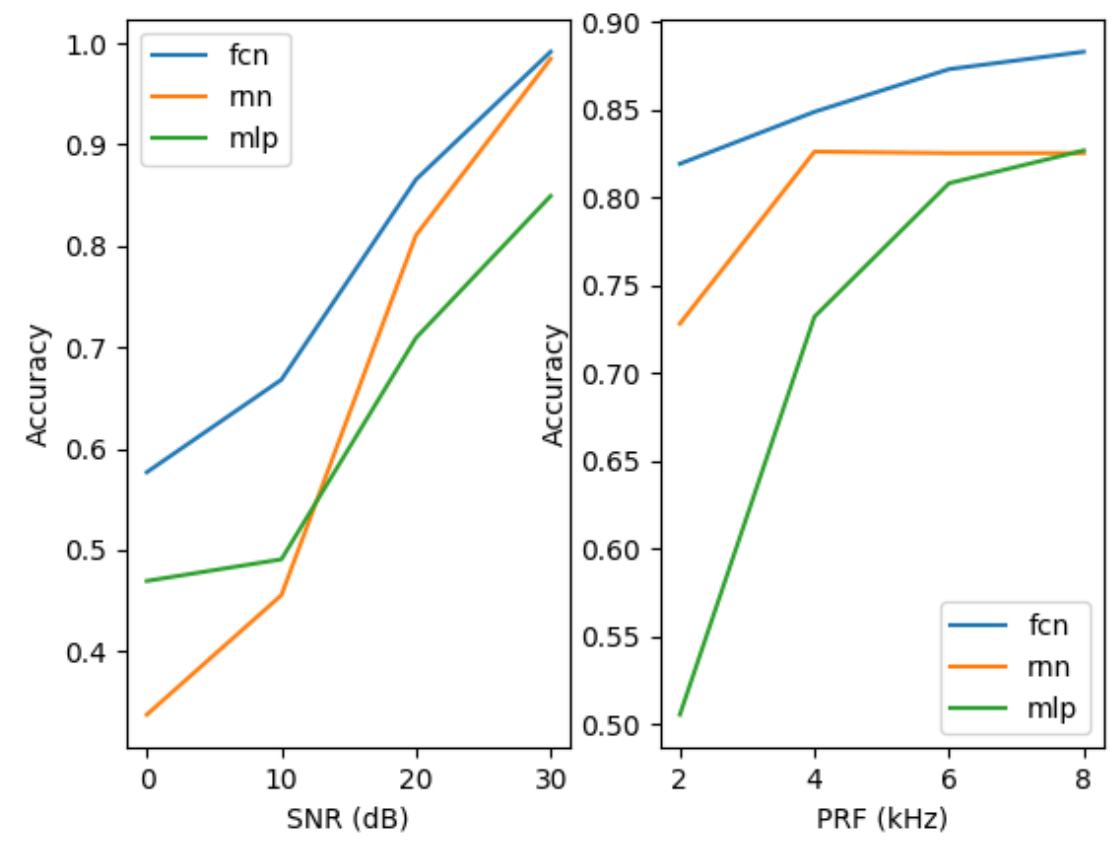

Figure 7: Performance of the learning models for increasingly good conditions. The graphs show intuitive behavior wrt the configurations.

lenging configuration being at $S N R=0 d B$ and $P R F=4 k H z$, we nevertheless achieve $\approx 58 \%$ with the FCN, versus total confusion $(\approx 33 \%)$ ) with the MLP. Note this is an unrealistically challenging configuration, as an $S N R$ of $0 d B$ means an original signal to noise ratio before Fourier transform of $\approx-15 d B$. Again, the FCN seems to be more robust to bad quality signals, although this should eventually be validated on real data. 


\section{Conclusion}

We have introduced an expressive simulator for blade-propelled engines such as drones with sufficiently realistic variational characteristics, which we have shown to be suited for machine learning on three distinct models: MLPs, RNNs and FCNs, which in turn we have experimentally validated to perform well even in harsh simulation conditions. We have furthermore justified the assumption that RNNs and FCNs that, because they can inherently learn temporal fluctuations, are particularly appropriate to the task of radar classification as they are able to handle signals of increasing length over time. Further developments for the simulator include its refinement to real-life subtleties and its extension to other kinds of UAVs. As for machine learning, the temporal architectures pave the way to more difficult tasks than classification, for instance detection, labelling and segmentation.

\section{References}

[1] M. Abadi, P. Barham, J. Chen, Z. Chen, A. Davis, J. Dean, M. Devin, S. Ghemawat, G. Irving, M. Isard, M. Kudlur, J. Levenberg, R. Monga, S. Moore, D. G. Murray, B. Steiner, P. Tucker, V. Vasudevan, P. Warden, M. Wicke, Y. Yu, and X. Zheng. TensorFlow: A System for Large-scale Machine Learning. In Proceedings of the 12th USENIX Conference on Operating Systems Design and Implementation, OSDI'16, pages 265-283, Berkeley, CA, USA, 2016. USENIX Association.

[2] D. K. Barton. Low-angle radar tracking. Proceedings of the IEEE, 62(6):687-704, June 1974.

[3] Y. Bengio, P. Simard, and P. Frasconi. Learning long-term dependencies with gradient descent is difficult. IEEE Transactions on Neural Networks, 5(2):157-166, Mar. 1994.

[4] V. Chen, F. Li, S.-S. Ho, and H. Wechsler. Analysis of micro-Doppler signatures. IEE Proceedings - Radar, Sonar and Navigation, 150(4):271, 2003.

[5] V. C. Chen, F. Li, S.-S. Ho, and H. Wechsler. Micro-Doppler effect in radar: phenomenon, model, and simulation study. IEEE Transactions on Aerospace and electronic systems, 42(1):2-21, 2006.

[6] F. Chollet et al. Keras. https: //keras.io, 2015.

[7] J. Collins, J. Sohl-Dickstein, and D. Sussillo. Capacity and Trainability in Recurrent Neural Networks. arXiv:1611.09913 [cs, stat], Nov. 2016. arXiv: 1611.09913.

[8] D. R. Cox. The regression analysis of binary sequences. Journal of the Royal Statistical Society. Series B (Methodological), pages 215-242, 1958.

[9] J. J. M. De Wit, R. I. A. Harmanny, and P. Molchanov. Radar micro-Doppler feature extraction using the singular value decomposition. In Radar Conference (Radar), 2014 International, pages 1-6. IEEE, 2014.

[10] T. Durand, T. Mordan, N. Thome, and M. Cord. WILDCAT: Weakly Supervised Learning of Deep ConvNets for Image Classification, Pointwise Localization and Segmentation. In IEEE Conference on Computer Vision and Pattern Recognition (CVPR 2017), Honolulu, HI, United States, July 2017. IEEE.

[11] S. Hochreiter and J. Schmidhuber. Long Short-Term Memory. Neural Comput., 9(8):1735-1780, Nov. 1997. 
[12] A. Krizhevsky, I. Sutskever, and G. E. Hinton. Imagenet classification with deep convolutional neural networks. In Advances in neural information processing systems, pages 1097-1105, 2012.

[13] L. a. B. Y. a. H. P. LeCun, Yann and Bottou. Backpropagation Applied to Handwritten Zip Code Recognition. 86(11):2278-2324.

[14] J. Long, E. Shelhamer, and T. Darrell. Fully convolutional networks for semantic segmentation. In Proceedings of the IEEE Conference on Computer Vision and Pattern Recognition, pages 34313440, 2015.

[15] P. Molchanov, R. I. Harmanny, J. J. de Wit, K. Egiazarian, and J. Astola. Classification of small UAVs and birds by micro-Doppler signatures. International Journal of Microwave and Wireless Technologies, 6(3-4):435-444, June 2014.

[16] F. Rosenblatt. The perceptron: A probabilistic model for information storage and organization in the brain. Psychological review, 65(6):386, 1958.

[17] S. Ruder. An overview of gradient descent optimization algorithms. arXiv:1609.04747 [cs], Sept. 2016. arXiv: 1609.04747.

[18] V. Schmidlin, G. Favier, R. Fraudt, and V. Schmidlin. Multitarget Radar Tracking with Neural Networks. IFAC Proceedings Volumes, 27(8):621-626, July 1994.

[19] Shannon C. E. A Mathematical Theory of Communication. Bell System Technical Journal, 27(3):379-423, July 2013.

[20] N. Takahashi, M. Gygli, and L. Van Gool. AENet: Learning Deep Audio Features for Video Analysis. arXiv:1701.00599 [cs], Jan. 2017. arXiv: 1701.00599.

[21] C. Tallec and Y. Ollivier. Can recurrent neural networks warp time? 